6-thioguanine (6-TGN) and 6-methylmercaptopurine (6-MMP). These metabolites, rather than the absolute dose of AZA, are associated with clinical efficacy and toxicity. An idiosyncratic skewed metabolism towards 6-MMP in some patients ("shunters") increases the risk of hepatotoxicity and treatment failure. Allopurinol can correct such shunts ${ }^{1}$. Therapeutic protocols using metabolite concentrations have been shown to be cost effective, improve efficacy, and decrease treatment morbidity in IBD' ${ }^{1}$. Only one small study estimated a therapeutic 6-TGN level for SLE patients ${ }^{2}$. There are scant data on AZA shunters in connective tissue disease $^{3}$.

Objectives: Explore the proportion of AZA therapeutic failure, toxicity, and shunters in CTD patients.

Methods: Retrospective, multicentre audit of AZA metabolite levels in CTD patients from 2012-2016. Patient demographics, treatments, disease activity and drug toxicity were also extracted.

Results: 61 testing episodes occurred in 34 patients whose mean age was 55 (32-79) years; predominantly female ( $\mathrm{N}=26,77 \%)$, with $\mathrm{SLE}(\mathrm{N}=19,56 \%)$. Active disease was present in 15/61 (25\%) episodes. 20/34 (60\%) patients were on HCQ + AZA. $25 / 34(76 \%)$ of patients were either on no or $<10 \mathrm{mg} /$ day of prednisone. 22/61 (36\%) episodes had bone marrow suppression, and 8/61 $(13 \%)$ had moderate liver function derangement. $12 / 35(34 \%)$ patients were AZA shunters.

Based on AZA metabolite levels, patients were classified into four categories:

\begin{tabular}{lccc}
\hline Classification $^{3}$ & 6-TGN & 6-MMP & Prevalence in Study (N) \\
\hline Underdosed/ noncompliant & Low & $\leq$ Normal & 9 \\
Appropriately dosed & Therapeutic & $\geq$ Normal & 23 \\
Overdosed & High & $\geq$ Normal & 16 \\
6-MMP Shunter & Low & $>$ Normal & 13 \\
\hline
\end{tabular}

SLE patients were over-represented in the underdosed/non-compliant category (7/9). Patients in underdosed and 6-MMP shunter categories had more active disease $(44 \%$ \& $31 \%)$, compared with appropriate and overdosed $(17 \%$ and $19 \% \mathrm{p}=0.24$ ). Surprisingly, patients who were overdosed by 6 TGN levels had the lowest mean AZA dose. 6-MMP shunters had significantly lower median 6TGN

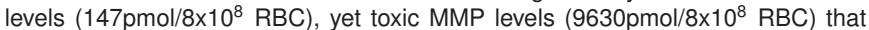
was associated with worst LFT derangement prevalence $(4 / 13,31 \%$ vs $<13 \%$, $\mathrm{p}=0.16)$.

Conclusions: AZA dose is poorly predictive of 6-TGN and 6-MMP levels. Overall, one third of CTD patients were shunters, with lower 6-TGN levels and more hepatotoxicity. Only $40 \%$ of test episodes were appropriately dosed. Patients with low disease activity are more likely to have adequate 6TGN levels, however, 40\% of patients with CTD won't have adequate disease control despite therapeutic 6-TGN levels. AZA metabolites can identify the multiple causes for therapeutic failure (under dosing, shunter, or true non-response), and enable early detection of shunters to avoid liver toxicity.

References:

[1] Croyle, L., \& Morand, E. F. (2015). Optimizing the use of existing therapies in lupus. Int J Rheum Dis 18(2), 129-37.

[2] Askanase, AD et al (2009). Use of pharmacogenetics, enzymatic phenotyping, and metabolite monitoring to guide treatment with azathioprine in patients with systemic lupus erythematosus. J Rheum, 36(1), 89-95.

[3] Haines, ML et al (2011). Clinical usefulness of therapeutic drug monitoring of thiopurines in patients with inadequately controlled inflammatory bowel disease. Inflam Bowel Dis, 17(6), 1301-7.

Disclosure of Interest: None declared

DOI: 10.1136/annrheumdis-2017-eular.2405

\section{AB0465 ASSOCIATION BETWEEN SAFETY, EFFICACY AND HYDROXYCHLOROQUINE DOSAGE IN THE TREATMENT OF CUTANEOUS LUPUS ERYTHEMATOSUS AND SYSTEMIC LUPUS ERYTHEMATOSUS}

R. Wakiya, T. Kameda, M. Izumikawa, S. Nakashima, H. Shimada, H. Ozaki, A. Kondo, N. Kadowaki, H. Dobashi. Department of Internal Medicine, Division of Hematology, Rheumatology and Respiratory Medicine, Kagawa University, Kagawa, Japan

Background: Hydroxychloroquine (HCQ) are considered to be effective against cutaneous lupus erythematosus (CLE) and symptoms associated with systemic lupus erythematosus (SLE) such as rashes, joint pain, and fatigue. In a randomized controlled trial in stable active patients with SLE on HCQ treatment, those who achieved blood HCQ levels greater than or equal to $1000 \mathrm{ng} / \mathrm{ml}$ had a tendency for reduced SLE flares during a 7 month period [1]. To prevent ocular toxicity, $\mathrm{HCQ}$ should be maintained at a dose of $6.5 \mathrm{mg} / \mathrm{kg}$ or less for ideal body weight [2], however, optimal HCQ dosage is unclear.

Objectives: To extract the problem of the dosage based on ideal body weight and identify safer and more effective $\mathrm{HCQ}$ dosage.

Methods: We enrolled patients who took HCQ for SLE or CLE more than 3 months in our institute and 2 related facilities from September 2015. We used Cutaneous Lupus Erythematous Disease Area and Severity Index (CLASI) to evaluate cutaneous symptoms and evaluated effect at start of administration and 3 months of that. The attending doctors assessed the adverse events (AEs). We investigated the change of serum biomarker, such as the value of serum complement and anti-ds-DNA body, the number of white blood cells, lymphocytes and platelets.

Results: We enrolled the 30 patients with administration of $\mathrm{HCQ}$ more than 3 months and in CLE were 2 cases, in SLE were 28 cases. In 21 cases HCQ dosage were based on ideal weight. The AEs were in $13 / 30$ cases (43.3\%). The AEs were the new cutaneous symptoms in 5 cases, eye manifestation in 3 cases (abnormal visual field in 2 cases, color anomaly in 1 case), diarrhea in 2 cases, fever in 2 cases, feeling of fatigue in 2 cases, renal dysfunction in 1 case, muscular pain in 1 case, and pericarditis in 1 case. The eye manifestation in the 3 cases disappeared for a few days by stopping or reducing $\mathrm{HCQ}$ dosage. Although we needed glucocorticoid treatment for pericarditis, the other AEs improved by reducing $\mathrm{HCQ}$ dosage or stopping. The AEs of taking HCQ $200 \mathrm{mg} /$ day were in 6 cases, $200 \mathrm{mg}$ and $400 \mathrm{mg}$ alternatively on every other day in 6 cases, and $400 \mathrm{mg} / \mathrm{day}$ in 1 case. The AEs of taking HCQ dosage based on ideal body weight were in 10/21 cases $(47.6 \%$ ) and by minimal dosage in $3 / 9$ cases (33.3\%). $22 / 28$ cases $(78.6 \%)$ significant improved cutaneous symptoms (amount of mean change of CLASI -4.57, $p=0.024)$. There is no difference in the efficacy of cutaneous symptoms between group received $\mathrm{HCQ}$ based on ideal body weight $(80.0 \%)$ and the others (92.3\%). 5/21 cases of HCQ based on ideal body weight were made to reduce HCQ dosage due to AEs, but all 5 cases improved cutaneous symptoms. 53.3\% $(16 / 30)$ cases increased complement, but other biomarkers didn't change.

Conclusions: $\mathrm{HCQ}$ was effective for the treatment of CLE even when $\mathrm{HCQ}$ dosage was reduced due to AEs. These findings suggest that low-dose HCQ is also effective and safe, and HCQ initial dosage wasn't the need to adjust for ideal body weight.

References:

[1] Costedoat-Chalumeau N, et al. Hydroxychloroquine in systemic lupus erythematosus: results of a French multicentre controlled trial (PLUS Study). Ann Rheum Dis. 2013;72(11):1786-92.

[2] Ronald B. Melles, Michael F.Marmor. The risk of toxic retinopathy in patients on long-term hydroxychloroquine therapy. JAMA Ophthalmol. 2014;132(12): 1453-60.

Disclosure of Interest: None declared

DOI: 10.1136/annrheumdis-2017-eular.3928

\section{AB0466 THE EFFECT OF OMEGA-3 FATTY ACIDS ON DISEASE ACTIVITY, ENDOTHELIAL FUNCTION, INFLAMMATORY MARKERS, AND LIPID PROFILE IN SYSTEMIC LUPUS ERYTHEMATOSUS: A SYSTEMATIC REVIEW AND META-ANALYSIS OF RANDOMIZED, CONTROLLED TRIALS}

R. Castillo ${ }^{1}$, P.P. Uy ${ }^{1}$, D.M. Francisco ${ }^{1}$, S. Lakshminarayanan ${ }^{2} .{ }^{1}$ Internal Medicine; ${ }^{2}$ Rheumatology, University of Connecticut, Farmington, United States

Background: Omega-3 fatty acids have been shown to have potentially beneficial immunomodulatory activity in autoimmune conditions such as rheumatoid arthritis and Sjogren's syndrome. Whether this extends to systemic lupus erythematosus (SLE) remains unclear.

Objectives: We undertook this study to summarize the body of evidence available from published clinical trials on the effectiveness of omega- 3 fatty acids on clinical and laboratory outcomes in SLE.

Methods: Two independent reviewers systematically searched PubMed, MEDLINE, Scopus, and the reference lists of related articles for studies published from inception to November 2016 using relevant keywords. Randomized, controlled trials (RCTs) on SLE patients comparing omega-3 fatty acids with placebo were included in the analysis. The quality of the included RCTs was assessed in accordance with the Cochrane Handbook. A random effects model was used to pool extracted data. Heterogeneity was evaluated with $\mathrm{Chi}^{2}$ test and $\mathrm{I}^{2}$, with $p$-values $<0.05$ considered significant. Data presented in median and interquartile range were converted to mean and standard deviation using the method described by Wan et al.

Results: A total of seven clinical trials consisting of 303 subjects with a duration of treatment ranging from 12 to 52 weeks were included. In studies using SLAM-R as the measurement of disease activity $(n=82)$, there was a statistically significant mean score reduction in the omega-3 fatty acid group vs. the placebo group at 24 weeks. However, in studies that used mean change in SELENA-SLEDAI $(n=117$, WMD: $\left.-0.87,95 \% \mathrm{Cl}:-3.9,2.17, \mathrm{I}^{2}=0 \%, \mathrm{p}=0.58\right)$ and PGA $(\mathrm{n}=117, \mathrm{WMD}-0.46$, $95 \% \mathrm{Cl}:-1.16,0.24, \mathrm{I}^{2}=85 \%, \mathrm{p}=0.20$ ) scores, there was no significant effect (Figure 1). Mean brachial artery diameter after 12 weeks likewise did not reveal

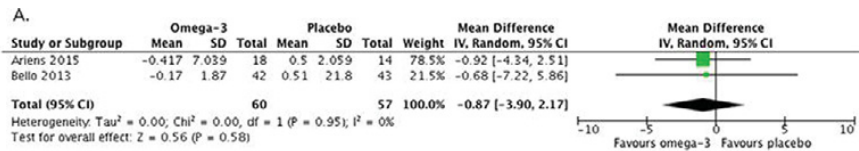

B.

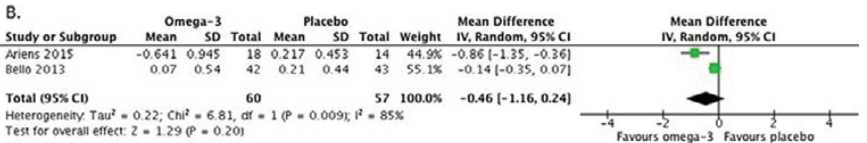

Figure 1. Effect of omega-3 fatty acids on SLE disease activity as measured by mean change in SELENA-SLEDAI (A) and PGA (B) scores. 